\title{
Several Forms of Li-Fan Integrable Unified Equation
}

\author{
Xingju Dang ${ }^{1}$, Yuhui $\mathrm{Luo}^{2}$ and Wenliang $\mathrm{Wu}^{3, *}$ \\ ${ }^{1,2,3}$ College of Physics \& Electronic Information Engineering, Zhaotong University, Zhaotong Yunnan, 657000, China \\ ${ }^{*}$ Corresponding author
}

\begin{abstract}
The naming the equation
$\mathrm{d} y / \mathrm{d} x+\{[(\mathrm{d} g / \mathrm{d} x) w+\mathrm{d} \psi / \mathrm{d} x] / g(x)-q(x) F[g(x) w(y)+\psi(x)]\} /(\mathrm{d} w / \mathrm{d} y)=0$

for Li-Fan Integrable Unified Equation is suggested. Several

forms of the equation are given.
\end{abstract}

Keywords-ordinary differential equation; nonlinear equation; first order equation; general integral; integrable unified equation

\section{INTRODUCTION}

In 1982, Prof. Hongxiang Li gave a first order differential integrable equation in [1]:

$$
y^{\prime}+P(x) y=y^{n} Q(x) F\left(y \mathrm{e}^{\int P(x) \mathrm{d} x}\right) .
$$

He pointed out that separable variable equation, linear equation, homogeneous equation and Bernoulli Equation are special cases of (1). In 1999, Prof. Li referred to (1) as unified equation of these integration equations in [2].

In 1987, Prof. Li and Xing Fan expanded (1) into the following form (Theorem 1.2 in [3]):

$$
\begin{gathered}
f(x) w^{\prime}(y) y^{\prime}+\left[P(x) f(x)+f^{\prime}(x)\right] w(y)= \\
{[f(x) w(y)+\varphi(x)]^{\alpha} \quad Q(x) F_{1}([f(x) w(y)+\varphi(x)] v(x))-} \\
P(x) \varphi(x)-\varphi^{\prime}(x),
\end{gathered}
$$

where

$$
v^{\prime}-P(x) v=k Q(x) v^{2-\alpha} .
$$

Its general integral is

$$
\int \frac{\mathrm{d} u}{u^{\alpha} F_{1}(u)+k u}=\int Q(x) v^{1-\alpha}(x) f(x) \mathrm{d} x+A .
$$

where $A$ is a arbitrary constant,

$$
u=v(x)[f(x) w(y)+\varphi(x)]
$$

In 1990, Prof. Li and Z.F.Starc discussed a class of more general first order ordinary differential integration equations, and gave some theorems and their corollaries in [4]. Equation (2) is mentioned as a corollary.

In order to facilitate the application, this paper simplifies (2), and give its several forms.

\section{THEOREM}

Suppose that $q, F \in \mathrm{C} ; g, w, \psi \in \mathrm{C}^{1} ; g(x) \neq 0$ and $w(y) \neq$ const, Equation

$y^{\prime}+\left(\frac{\mathrm{d} w}{\mathrm{~d} y}\right)^{-1}\left\{\frac{g^{\prime}(x) w(y)+\psi^{\prime}(x)}{g(x)}-q(x) F[g w+\psi]\right\}=0$

can be integrated. Its general integral is

$$
\int \frac{\mathrm{d} u}{F(u)}=\int g(x) q(x) \mathrm{d} x+C
$$

where

$$
u=g(x) w(y)+\psi(x)
$$

$C$ is a arbitrary constant.

\section{PROVE}

Let $u=g(x) w(y)+\psi(x)$, then

$$
u^{\prime}=g^{\prime}(x) w(y)+g(x) w^{\prime}(y) y^{\prime}+\psi^{\prime}(x)=
$$

$$
\begin{gathered}
g(x)\left\{q(x) F[g(x) w(y)+\psi(x)]-\frac{\psi^{\prime}(x)}{g(x)}\right\}+\psi^{\prime}(x)= \\
g(x) q(x) F[g(x) w(y)+\psi(x)]=g(x) q(x) F(u) .
\end{gathered}
$$

It is a separable equation and its general solution is (7). Then the theorem is proved.

$$
\begin{aligned}
& \text { Let } g(x)=v(x) f(x), \psi(x)=v(x) \varphi(x), \\
& q(x)=[v(x)]^{\alpha} Q(x), F(u)=u^{\alpha} F_{1}(u)+k u,
\end{aligned}
$$


if the requirement (3) was founded, then (6) becomes (2), and (7) becomes (4).

\section{COROLlaries}

Corollary 1 Suppose that $P, q, F \in \mathrm{C} ; w, \psi \in \mathrm{C}^{1} ; w(y) \neq$ const. Equation

$$
y^{\prime}+\frac{P(x) w(y)+\psi^{\prime}(x) \mathrm{e}^{-\int P(x) \mathrm{d} x}-q(x) F\left[w \mathrm{e}^{\int P(x) \mathrm{d} x}+\psi\right]}{\mathrm{d} w / \mathrm{d} y}=0
$$

is integration.

Proof In (6), taking $g(x)=\mathrm{e}^{\int P(x) \mathrm{d} x}$, (6) becomes (9). According to Theorem the proof is ended.

Corollary 2 Suppose that $f, q, F \in \mathrm{C} ; g, \quad \psi \mathrm{C}^{1} ; g(x) \neq 0$. Equation

$$
y^{\prime}=\frac{g^{\prime}(x)}{g(x)} f(y)+f(y) \mathrm{e}^{\int \frac{\mathrm{d} y}{f(y)}}\left\{\frac{\psi^{\prime}(x)}{g(x)}-q(x) F\left[g(x) \mathrm{e}^{-\int \frac{\mathrm{d} y}{f(y)}}+\psi\right]\right\}
$$

is integration.

Proof In (6), taking $w(y)=\mathrm{e}^{-\int \frac{1}{f(y)} \mathrm{d} y},(6)$ becomes (10). According to Theorem the proof is ended.

Corollary 3 Suppose that $P, q, f, F \in \mathrm{C} ; \psi \in \mathrm{C}^{1}, f(y) \neq 0$. Equation

$$
\begin{gathered}
y^{\prime}=P(x) f(y)+f \exp \left(\int \mathrm{d} y / f\right)\left\{\psi^{\prime}(x) \exp \left(-\int P \mathrm{~d} x\right)-\right. \\
\left.q(x) F\left[\exp \left(\int P \mathrm{~d} x-\int \mathrm{d} y / f\right)+\psi\right]\right\}
\end{gathered}
$$

Is integrable.

Proof In (6), taking $w(y)=\mathrm{e}^{-\int \frac{1}{f(y)} \mathrm{d} y}, g(x)=\mathrm{e}^{\int P(x) \mathrm{d} x}$, (6) becomes (11). According to Theorem the proof is ended.

We suggest the naming (5), (9),(10) and (11) for Li-Fan Integrable Unified Equations when the requirements in the theorem or corollarys was founded.

Corollary 4 Suppose that $P, q, F \in \mathrm{C}, \psi \in \mathrm{C}^{1}$. Equation

$$
y^{\prime}=P(x) \pm \mathrm{e}^{ \pm y}\left\{\psi^{\prime}(x) \mathrm{e}^{-\int P \mathrm{~d} x}-q(x) F\left[\mathrm{e}^{\int P \mathrm{~d} x \mp y}+\psi\right]\right\}
$$

is integrable.

Proof In (11), let $f(y) \equiv \pm 1$, if $f(y) \equiv-1$, then $P(x)$ is replaced by $-P(x),(11)$ becomes (12). According to Corollary 3 the proof is ended.
Corollary 5 Suppose that $P, q, F \in \mathrm{C} ; \psi \in \mathrm{C}^{1}$. Equation

$$
y^{\prime}=P(x) y+y^{2}\left\{\psi^{\prime}(x) \mathrm{e}^{-\int P \mathrm{~d} x}-q(x) F\left[\frac{\mathrm{e}^{\int P \mathrm{~d} x}}{y}+\psi\right]\right\}
$$

is integration.

Proof In (11), let $f(y)=y,(11)$ becomes (13). According to Corollary 3 the proof is ended.

Corollary 6 Suppose that $P, q, F \in \mathrm{C}, \psi \in \mathrm{C}^{1}$. Equation

$$
y^{\prime}=-P(x) y+\left\{\psi^{\prime}(x) \mathrm{e}^{-\int P \mathrm{~d} x}-q(x) F\left[y \mathrm{e}^{\int P \mathrm{~d} x}+\psi\right]\right\}
$$

is integration.

Proof In (11), let $f(y)=-y,(11)$ becomes (14). According to Corollary 3 the proof is ended.

In (14), let $\psi(x) \equiv 0, q(x)=Q(x) \exp \left(n \int P(x) \mathrm{d} x\right), F(u)$ is replaced by $F(u) u^{-n},(14)$ becomes (1).

Corollary 7 Suppose that $q, \Phi \in \mathrm{C} ; m, n \in \mathrm{C}^{1}$. Equation

$$
y^{\prime}=-\frac{m^{\prime}(x) y+n^{\prime}(x)}{m(x)}+q(x) \Phi[m(x) y+n(x)]
$$

is integration.

Proof In (6), let $w(y)=y, g(x)=m(x), \psi(x)=n(x), F(u)=\Phi(u)$, (6) becomes (15). According to Theorem the proof is ended. In fact, this corollary is Theorem in [5] by Prof. Luxiang Feng in 2013 .

Corollary 8 If equation

$$
y^{\prime}-a \lambda(x) y=q(x) \Phi[(b y+c) G(\lambda)]+\frac{a c}{b} \lambda
$$

meet requirement $\left[\frac{1}{G(\lambda)}\right]^{\prime}-\frac{a \lambda}{G}=k q$, then it is integration.

Proof In (2), let $w(y)=b y+c, g(x) \equiv 1, \varphi(x) \equiv 0, v(x)=G(\lambda(x))$, $F_{1}(u)=\Phi(u), P(x)=-a \lambda(x), \alpha=0,(3)$ is met. (2) becomes (16). According to the prove of Theorem the proof is ended. In fact, this corollary is Theorem in [6] by Prof. Feng in 2012 ( $d$ is replaced by $k$ here).

Corollary 9 Equation

$$
y^{\prime}-\frac{h^{\prime}}{h} y=q \Phi\left[\frac{y+f}{h}\right]+\frac{h^{\prime}}{h} f-f^{\prime}+\alpha q
$$

is integration. 
Proof In (6), let $w(y)=y, g(x)=1 / h(x), \psi(x)=f(x) / h(x)$, $F(u)=\Phi(u)+\alpha$, then

$$
\frac{g^{\prime}}{g}=h\left(\frac{1}{h}\right)^{\prime}=-\frac{h^{\prime}}{h} .
$$

(6) becomes (17). According to Theorem the proof is ended. In fact, this corollary is Theorem in [7] by Prof. Zhilin Li in 2009 ( $g$ is replaced by $h$ here).

Corollary 10 Equation

$$
y^{\prime}=p(x) y+Q(x)(y+f(x))^{n}+p(x) f(x)-f^{\prime}(x)
$$

is integration.

Proof In (9), let $\quad w(y)=y, \quad P(x)=-p(x)$, $g(x)=\exp \left(-\int p(x) \mathrm{d} x\right), q(x)=Q(x) \exp \left(n \int p(x) \mathrm{d} x\right)$, $\psi(x)=f(x) \exp \left(-\int p(x) \mathrm{d} x\right), F(u)=u^{n}$, then

$$
\begin{gathered}
\psi^{\prime}(x) \exp \left(-\int P(x) \mathrm{d} x\right)=\psi^{\prime}(x) \exp \left(\int p(x) \mathrm{d} x\right)= \\
{\left[\psi(x) \exp \left(\int p(x) \mathrm{d} x\right)\right]^{\prime}-\psi(x) \exp \left(\int p(x) \mathrm{d} x\right) p(x)=} \\
f^{\prime}(x)-p(x) f(x) .
\end{gathered}
$$

(9) becomes (18). According to Corollary 1 the proof is ended. In fact, this corollary is Theorem 1 in [8] ( $q$ is replaced by $Q$ here).

\section{REFERENCES}

[1] Hongxiang Li. "Elementary quadratures of ordinary differential equations", in Amer Math Monthly, vol 89, pp.:198-208, March 1982.

[2] Hongxiang Li. "Unified equation and unified transformation on several classes of first order differential equation", in Studies In College Mathematics, pp.11-14. April 1999.

[3] Hongxiang Li, and Xing Fan. "Re-discussion on elementary quadratures of ordinary differential equations", in J. Shanghai Inst. of Railway Tech, vol. 8, pp.1-15. June 1987.

[4] Hongxiang Li, and Zdravko F Starc. "Several Classes of Integrable Nonlinear Ordinary Differential Equations(I) First-Order Equations", in Applied Mathematics and Mechanics, vol. 11, pp.:561-560. March 1990.

[5] Luxiang Feng. "Integrability Condition and Its Application of the First Order Generalized Ordinary Differential Equation", in J. Yunnan Normal University, vol. 33, pp.:25-28. July, 2013.

[6] Luxiang Feng. "A Class of First Order Differential Equation and Application of the Integrability Conditions", in J. MUC (Natural Sciences Edition) Normal University, vol. 21, pp.:32-36. February, 2012.

[7] Zhilin Li. "A Note on the Integrability Conditions of Nonlinear Differential Equation of the $1^{\text {st }}$ Kind", in Henan Science, vol. 27, pp.:906-908. August, 2009.

[8] Le Yi. "Integrability conditions and general solutions of a kind of first order nonlinear differential equation", in J. Guizhou Normal University (Natural Sciences). Vol. 29, pp.:91-92. May, 2011. 